\title{
Pigmented Skin Lesions Studied by VIS-NIR Diffuse Reflectance Spectroscopy
}

\author{
M. Cordo Chinea*a,b J.R. Sendra Sendra ${ }^{\text {a,b }}$, S.M. López Silva ${ }^{a, b}$, A. Viera Ramírez \\ ${ }^{a}$ IUMA, University of Las Palmas de Gran Canaria, Las Palmas de Gran Canaria 35017, Spain; \\ ${ }^{\mathrm{b}}$ ICIC, Instituto Canario de Investigacion del Cancer, Spain; \\ ${ }^{\mathrm{c}}$ Dermocanarias Medico-Quirurgica S.L., Las Palmas de Gran Canaria, Spain
}

\begin{abstract}
Pigmented skin lesions have been studied by optical diffuse reflectance spectroscopy. Our measure system consists of a portable visible near infrared $(550-1000 \mathrm{~nm})$ spectrometer, tungsten-halogen lamp and fibre optic probes. The system was tested in steady state conditions. After that, a reproducibility study of normal and pigmented skin spectra was carried out. A small scale study has been conducted in human volunteers with different clinically evaluated lesions. The analysis of the collected spectra is shown.
\end{abstract}

Key Words: optical spectroscopy, reflectance spectroscopy, skin pigmented lesions, fibre optics.

\section{INTRODUCTION}

The incidence of pigmented skin lesions in the population has increased in the last decades ${ }^{1-4}$. An early detection of malignant lesions, such as the melanoma, gives the patient the best chance of cure and allows a mortality reduction. The clinical diagnosis of melanoma is based on the visual features of the lesion, the so-called ABCDE system (asymmetry, border, colour, dimension and evolution of the lesion) ${ }^{2}$. As result of the difficulties in achieving an early diagnosis by visual assessment, there stays the need for surgical biopsies and histopathological analysis for the majority of benign lesions.

Several studies using spectrophotometric techniques ${ }^{5-12}$ have been undertaken in an effort to sharpen the dermatological diagnostic criteria. In general, these researches have found evidences of spectrophotometric differences between benign and malignant lesions. Among the diagnosis tools developed to aid the dermatologist to distinguish between malignant and non malignant lesions, dermatoscopy (epiluminiscense microscopy, dermoscopy, oil immersion microscopy, skinsurface microscopy) allows the visualisation of morphological characteristics at the dermoepidermal junction using oil and a hand-held microscope $\mathrm{e}^{12}$. Other optical spectroscopy based technique ${ }^{13-15}$ provides spectra that contain information about the morphology of the tissue as well as the chromophore content and has been applied to study benign pigmented lesions and melanoma ${ }^{14}$.

The aim of this work is to study the optical diffuse reflectance spectra of pigmented skin lesions using a fibre optics probe coupled to a visible near infrared (VIS-NIR) spectrometer and to evaluate the recorded spectra features for improving the differential diagnosis of healthy skin and pigmented cutaneous lesions. First, the parameters of our measure system and their variability have been tested in steady state conditions. After that, a reproducibility study of both normal and pigmented skin diffuse reflectance spectra has been carried out. Once established the optimal working parameters for our measure system, a small-scale study of clinically evaluated skin pigmented lesions have been conducted. Here we expose the preliminary results obtained in this small-scale study.

*mcordo@iuma.ulpgc.es; phone +34 928 457330; fax +34 928451243 


\section{MATERIALS AND METHODS}

\subsection{Measure System}

The experimental setup used to acquire reflectance spectra from skin lesions is shown in Figure 1. The measure system consists of a light source, fibre optics reflection probe, a portable VIS-NIR spectrometer and a portable personal computer. The light source is an Avantes HL-2000 miniature stabilized tungsten-halogen fan-cooled lamp with a filter slot that accepts 2', 2 '"square filters. We use in our experiments reflection probes (Avantes) either standard type (FCR-7IR200-2) or with a small tip (FCR-7IR200-2-1.5x100). The probe ends are made of stainless steel, being their external diameter $6.35 \mathrm{~mm}$ (FCR-7IR200-2) and $1.5 \mathrm{~mm}$ (FCR-7IR200-2-1.5x100) respectively. Both reflection probes have one central fibre surrounded by six fibres. The seven individual fibres are $200 \mu \mathrm{m}$ in total diameter with numerical aperture 0.22 and transmission in the spectral range from visible to near infrared (350-2000 nm). Via a standard SMA905 connector, the light from the source is coupled into the central fibre, which transmits the optical radiation to the skin. The six fibres collect the light reflected from the skin.

To minimize the external light interference both types of probe ends are covered with a black protector once placed in contact with the region of the skin under investigation. Special care is taken to avoid the application of pressure or deformation and vascularization changes in the skin zone analysed. The six reflected light collecting fibres are coupled to the "Avantes AVS-USB 2000" spectrometer input using a standard SMA905 connector. The selected spectrometer grating allows covering the spectral range from $530-1100 \mathrm{~nm}$. The spectrometer output is connected to the computer via the Universal Serial Bus (USB) port. The "Avantes AvaSoft-Full Version" software permits to control the measure system parameters and optical spectra recording.

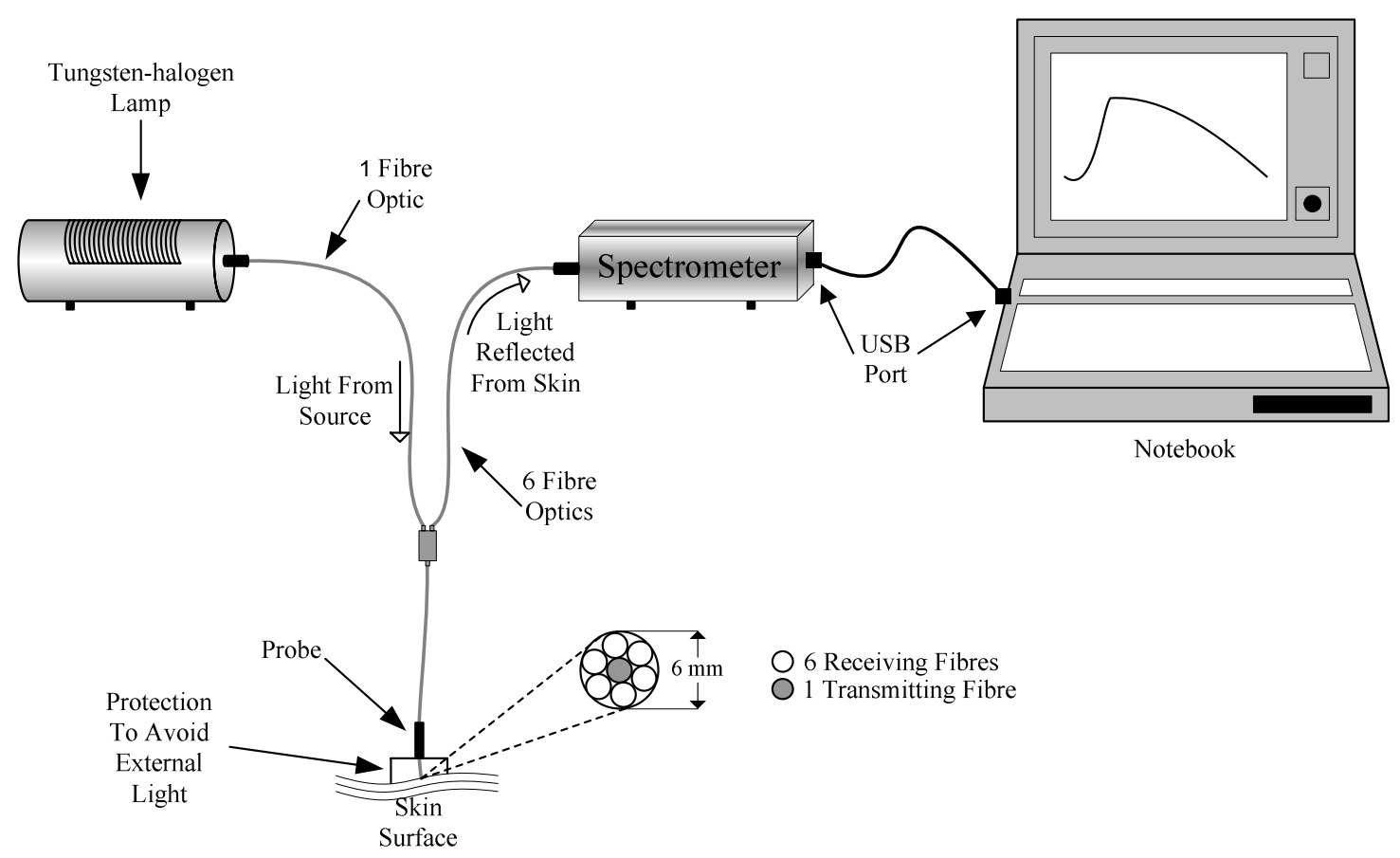

Figure 1. Experimental setup used to acquire reflectance spectra from skin. 


\subsection{Measure System Reproducibility and Parameters}

The spectral signal for reflected light is expressed in terms of percentage reflectance regarding white reference after dark correction as:

$$
\mathrm{R}(\lambda)=\frac{\mathrm{S}(\lambda)-\mathrm{D}}{\operatorname{Ref}(\lambda)-\mathrm{D}}
$$

where $S(\lambda)$ is the spectral signal for light reflected from the analyzed medium at each wavelength $\lambda$, D is the dark signal and $\operatorname{Ref}(\lambda)$ is the white reference one. The dark signal is measured by closing the light output from the lamp to the illumination fibre using a shutter, a black metal sheet introduced in the lamp filter slot, while the probe is kept in contact with the analyzed medium (the skin in our case). Thus D results from the detector dark current and also from all ambient light that reaches the collection fibres (e.g. ambient light re-emitted by the skin). The reference, defined as $100 \%$ reflectance at all wavelengths, is measured by placing the probe in contact with a white tile based on diffused Teflon (WS-2, Avantes). In this way the $\operatorname{Ref}(\lambda)$ signal includes all effects of light source, optical fibres and any other background excluded from the analyzed medium. The light source output is attenuated when needed, using a neutral filter (GL-NG9-1, Avantes). The average of several readings, computed in real time, can be stored for each spectral measurement.

A reproducibility study was conducted in order to check our system performance and to define the measurement parameters. The system was tested in steady state conditions by using a GL-NG9-1 neutral filter and the white reference tile WS-2, for different values of Integration Time (IT=3-6 ms) and Spectra Averaging (AV=2-50). As the light source time to stabilize is $5 \mathrm{~min}$, the lamp was switched on $15 \mathrm{~min}$ before the making of any reflectance measurements $(\mathrm{S}(\lambda))$ at each wavelength $(\lambda)$. The analysis of the recorded 1000 spectra let to obtain its mean, Standard Deviation (SD), variance, $\log$ SD vs. $\log \mathrm{AV}$ and to consider the optimal values of IT and AV. The number of measurements averaged is chosen as a trade off between reduction of spectral noise and sampling time. The optimal values were 40 for AV and 30 $\mathrm{ms}$ for IT. The integration time was reduced to $6 \mathrm{~ms}$ due to the saturation of the spectral signal in the case of the white reference. Further to it, measurements of D had exhibited no measurable dependence on wavelength and a slight dependence on IT.

To study the reproducibility of skin reflectance spectra measurements a series of $3 \mathrm{~S}(\lambda)$ readings were taken on 10 different days from 4 sites on a female volunteer: normal skin on the outside upper arm, normal skin on the inside upper arm, a lesion (naevus intradermal) on the neck and skin adjacent to the lesion. Each reading was the averaging of 10 averaged measurements. In this case, the measure system parameters set as AV=10 and $30 \mathrm{~ms}$ for $\mathrm{S}(\lambda)$ IT and $6 \mathrm{~ms}$ for $\operatorname{Ref}(\lambda)$ IT. D and $\operatorname{Ref}(\lambda)$ were measured in every session before and after, respectively, each $S(\lambda)$ measurement. The lamp was switched on 15 min before the making of any reflectance measurements $(S(\lambda))$ at each wavelength $(\lambda)$. The dark (D) and reference $(\operatorname{Sref}(\lambda))$ measurements were repeated immediately before each skin measurement. The white tile WS-2 (Avantes) based on diffuse Teflon was used as the reflectance standard or reference. The light source output was filtered, when it was needed, using a neutral filter (GL-NG9-1, Avantes). In the Figure 2 are shown the resulting mean spectra $+/$ - one standard deviation calculated from the total of the readings according to expression (1). The sources of variations between the readings were probably the variations in blood content, pressure of the probe on the surface of the skin, instability of the lamp output and difficulty in repeating probe position on the skin surface, especially in the lesion, due to the heterogeneous of the skin.

\subsection{Clinical Patient Selection and Data Acquisition}

Once established the optimal working parameters for our measure system, we proceeded to study skin lesions spectra according to the flow diagram for the application of diffuse reflectance spectroscopy to dermatological diagnosis shown in Figure 3. A small-scale preliminary study of clinically diagnosed skin pigmented lesions has been conducted to evaluate the possibilities of our measure system to record reflectance spectra from different lesions and the reproducibility of these spectra. 


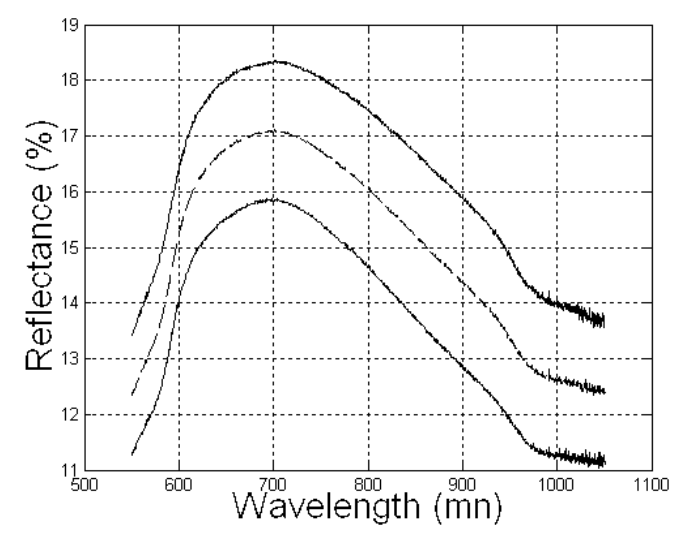

(a)

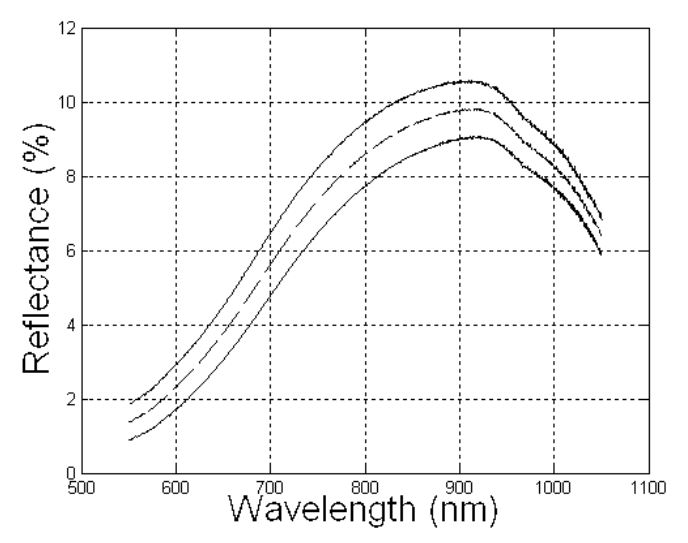

(c)

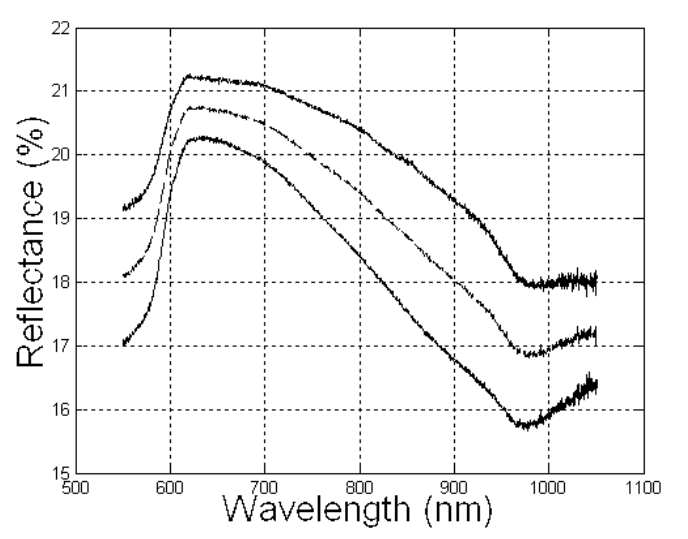

(b)

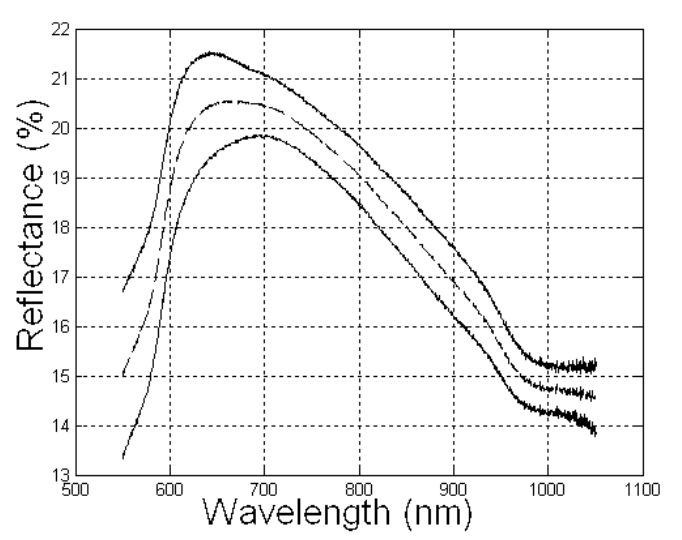

(d)

Figure 2. Mean (central curve) and $+/$ - one standard deviation of (a) normal skin in the outside upper arm, (b) normal skin in the inside upper arm, (c) the lesion itself (naevus) on the neck and (d) skin adjacent to the lesion.

The study of skin lesions was performed on 17 lesions from 15 patients - volunteers, ranging in age from 24 to 43 years old. Informed consent was obtained from each patient before any measurement. All lesions were evaluated by the dermatologist using both visual analysis and epiluminiscence microscopy. In Table 1 are summarized the clinical diagnosis of the selected lesions and its location. The lesions were defined as benign (histological diagnosis was not required), suspicion of malignancy or malignant. All of them were included in the reflectance spectroscopy study because they exhibited differences in morphological patterns. Only two lesions had morphological and epiluminiscent atypical signs and were due to be excised on suspicion of malignancy. The histological examination has not yet been completed and it will bring the final diagnosis about the malignancy of these two lesions.

After the definition of the lesions clinical diagnosis, the diffuse reflectance measurements were performed as shown on the right side of the flow diagram in Figure 3. The reflectance spectra were collected for all patients and lesions according to the established protocol from the following sampling areas: 


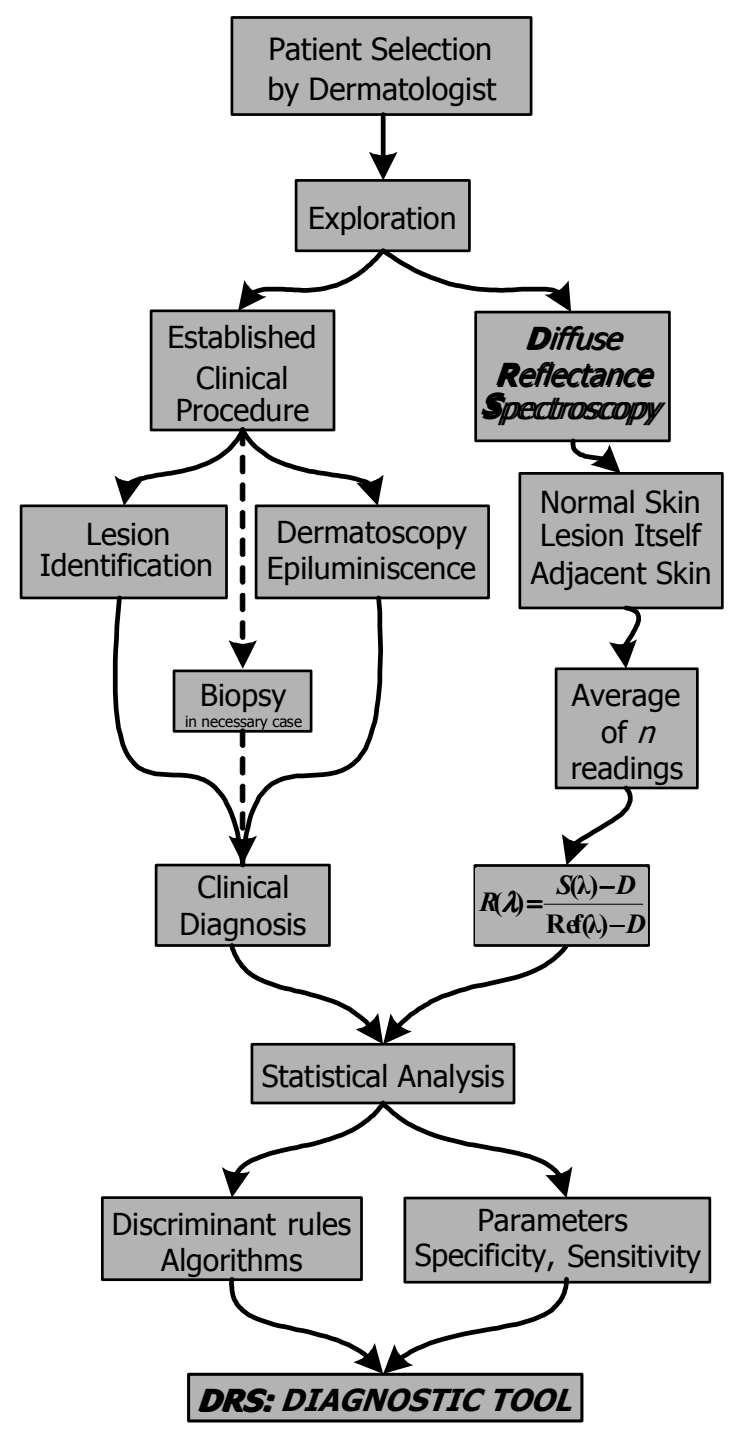

Figure 3. Flow diagram for applying diffuse reflectance spectroscopy to dermatological diagnosis.

(I) Healthy skin in the outside upper arm, a region defined as skin that is normally exposed to sun light (tanned). Three spectra were obtained from that region.

(II) Healthy skin in the inside upper arm, a region defined as skin that is not normally exposed to sun light (not tanned). Three spectra were obtained from that region.

(III) Lesion: we measured three reflectance spectra from the lesion itself. In some cases the dermatologist indicated the right place inside the lesion where the reflectance spectra should be measured, and sometimes, as in the case of the naevus clinically atypical and others, we measured more than one location inside the lesion. Depending in the lesion dimensions and characteristics we used the tip probe for a more precise measurement.

(IV) Skin surrounding the lesion: three spectra were measured in the skin adjacent to the lesion on the vertexes of an imaging equilateral triangle, one on the top of the lesion and the other surrounding it. 
Thus we could verify similarities and disparities between the reflectance spectra from the area surrounding the lesion and the outside upper arm, for a lesion usually exposed to sunlight. It is due to the fact that skin adjacent to the lesion could be more pigmented than normal skin. If the lesion is on an area that is not exposed to sunlight, we could verify similarities with healthy skin in the inside upper arm.

In each region, the final spectrum was the average of three of them. The lamp was ignited 30 min before making any reflectance measurements. For each spectra measurement the average of 40 readings was stored. The reference signal was recorded before each measurement on each patient and on every region having IT $=6 \mathrm{~ms}$. Then, we changed the integration time to $30 \mathrm{~ms}$ and placed the probe against the skin we wanted to measure. Once the spectrum was stable we proceeded to record three of them. After that and without moving the probe, we took the dark signal by closing the output of the light from the lamp with the external shutter. After all the measurement sessions, we processed all the signals to obtain the reflectance spectra from four regions in each patient.

Table 1. Lesions studied, listed by clinical type.

\begin{tabular}{|c|c|c|c|c|c|}
\hline Lesion No. & Lesion Clinical Diagnosis & Patient & $\begin{array}{c}\text { Patient } \\
\text { Lesion }\end{array}$ & $\begin{array}{c}\text { Female/ } \\
\text { Male }\end{array}$ & Localization \\
\hline 1 & Naevus nevocitic benign & P01 & L01 & M & Forehead \\
\hline 2 & Naevus nevocitic benign congenital & P02 & L01 & M & Neck \\
\hline 3 & Naevus nevocitic intradermal benign & P03 & L01 & M & Knee \\
\hline 4 & Naevus junctional benign & P04 & L01 & F & External arm \\
\hline 5 & Angioma & P04 & L02 & F & Chest \\
\hline 6 & Naevus nevocitic benign dermoepidermal & P04 & L03 & F & Back \\
\hline 7 & Efelide & P05 & L01 & M & Back \\
\hline 8 & Dermatofibroma & P06 & L01 & M & Neck \\
\hline 9 & Naevus nevocitic (slightly atypical-5 mm) & P07 & L01 & F & Abdomen \\
\hline 10 & Naevus nevocitic dermoepidermal & P08 & L01 & M & Chest \\
\hline 11 & Naevus dermoepidermal & P09 & L01 & M & Forehead \\
\hline 12 & Efelide & P10 & L01 & F & External arm \\
\hline 13 & Naevus dermoepidermal & P11 & L01 & M & Throat \\
\hline 14 & Naevus clinically atypical $(8.5$ mm) & P12 & L01 & M & Back \\
\hline 15 & Lentigo & P13 & L01 & M & Back \\
\hline 16 & Naevus nevocitic intradermal benign & P14 & L01 & M & Chin \\
\hline 17 & Angioma & P16 & L01 & M & Chest \\
\hline
\end{tabular}

\section{RESULTS}

\subsection{Reflectance Spectra}

The goal of this study was to obtain the optical reflectance spectra of pigmented skin lesions and healthy skin. In Figure 4 are shown the reflectance spectra from six of the cases in Table 1. The number after the L, in the caption of Figure 4 , corresponds to the lesion number in the first column of Table 1. Some of the lesions listed in Table 1 have been excluded since the spectra were not clear and not repetitive due to the presence of hair.

Visual analysis shows that the values for the reflectance spectra of clinically atypical and slightly atypical lesions are significantly different from those of other pigmented lesions (the non-malignant lesions). The slope in the reflectance spectra of the non-malignant lesions is steeper than the slope in the suspicious of malignancy between $550-800 \mathrm{~nm}$. The values for the reflectance are higher in the usual lesions (i.e. the non-malignancy lesions) and the reflectance in the atypical lesions is no more than $11 \%$ in the two cases studied. The case of the angioma is the easiest to differentiate 
since it distinctly shows feature due to oxyhaemoglobin absorption (around $574 \mathrm{~nm}$ ) but this lesion is not defined as a pigmented lesion, so it was excluded for further analysis.

The non-malignant lesions (i.e. efelide, dermatofibroma, naevus nevocitic intradermal and lentigo) have a maximum peak of reflectance between 650 and $850 \mathrm{~nm}$, while in the case of the suspicious of malignancy the peak of reflectance is around $850-950 \mathrm{~nm}$. After this maximum of reflectance, the slope of the spectra in all of the non malignant lesions is negative and fall off to reflectance values, in the range of 900-1000 nm, similar to the values in the 550-600 nm range. Nevertheless the malignant lesions have also a negative slope in the reflectance spectra above $900 \mathrm{~nm}$, but is smoother than the non malignant and do not fall off as they do.

Despite the efelide is a non malignant pigmented lesion, its spectra (Figure 4 (b)) is very similar to the spectra of the naevus clinically atypical (Figure 4 (f)), with a higher value of the maximum reflectance (about 13.5\%). Since the efelide is a smooth, flat, with border regularity, and small diameter lesion, it morphologically differs from any malignant lesions and can be distinguish from the other by visual analysis. Although both spectra are similar, the lesions are clinically different.

The dark color of the atypical lesions considered in this study, with high concentration of melanin on it, gives to these lesions small reflectance values between $550-800 \mathrm{~nm}$. In this wavelength range the reflectance values are around $2 \%$ in $550 \mathrm{~nm}$ and rise until 10\% around $800 \mathrm{~nm}$. However, in the non malignant lesions the values for the reflectance in that wavelength range are higher, starting with a value nearest $10 \%$ at $550 \mathrm{~nm}$ and reaching the highest values around 750 $800 \mathrm{~nm}$, with reflectance peaks of $11.5-14.5 \%$.

\subsection{Normalized Reflectance Spectra}

In order to follow the work started by Marchesini et $\mathrm{al}^{5}$ and continued by Wallace et $\mathrm{al}^{11}$, we normalized all the lesion spectra by the spectra from the healthy skin. This normalized spectrum was calculated by dividing the lesions reflectance $\left(\mathrm{R}_{\mathrm{L}}(\lambda)\right.$ ) data (area III in 2.3) by those of the healthy skin. In our study the spectra of healthy skin were collected from three different places: outside upper arm (area I in 2.3), inside upper arm (area II in 2.3), and skin surrounded the lesion (area IV in 2.3). Marchesini et $\mathrm{al}^{5}$ found that differences extracted from lesions were correlated with the features associated with healthy adjacent skin. In our research we took into account this normalized spectra to find similarities between the spectra from the lesion divided by the spectra of the outside upper arm $\left(\mathrm{R}_{\mathrm{L}}(\lambda) / \mathrm{R}_{\mathrm{OA}}(\lambda)\right)$ and the spectra from the lesion divided by the spectra of the adjacent skin $\left(R_{L}(\lambda) / R_{L S}(\lambda)\right)$ in the case of lesion exposed to the sun light (i.e. head, external arm, back in people who work out). On the other hand, we expected to find similarities in the lesion spectra divided by the spectra of the inside upper arm $\left(R_{L}(\lambda) / R_{I A}(\lambda)\right)$ and the spectra from the lesion divided by the spectra of the adjacent skin $\left(\mathrm{R}_{\mathrm{L}}(\lambda) /\left(\mathrm{R}_{\mathrm{LS}}(\lambda)\right)\right.$ in the case that the lesion was in a place not exposed to the sun light.

It is early to know if this analysis will be useful to determine differences between benign and malignant lesions. An abnormal longitudinal growth of the lesion would involve a variation in the melanin content of the original surrounded skin, so a different spectrum would appear. Using the epiliminiscense microscopy the specialist could analyse the change under the epidermis, but sometimes this change is not clear by visual assessment. In this case the study of the normalized spectra could predict this subtle difference.

In this preliminary study with a non-statistically significant number of patients and lesions, the individual analysis showed no differences for the three types of normalization used. It seems to be related with the non-exposure to the sun, a common characteristic of the group studied. Nevertheless we should verify this in a larger group of patients and lesions. 


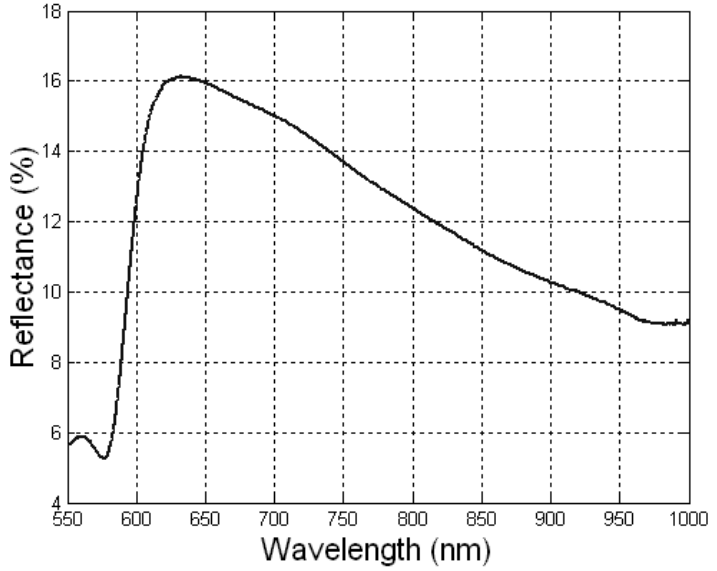

(a)

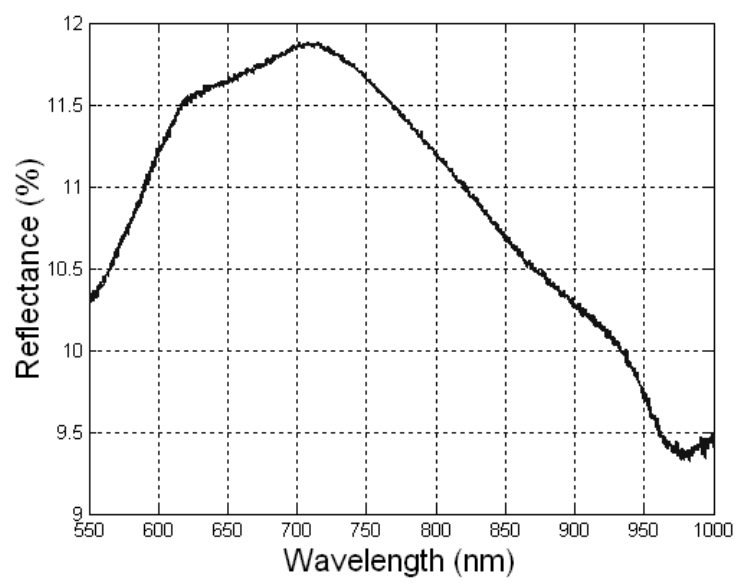

(c)

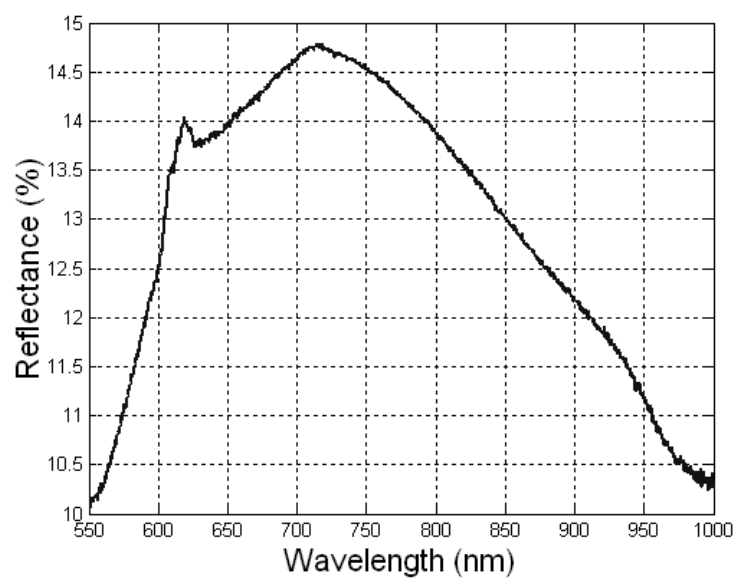

(e)

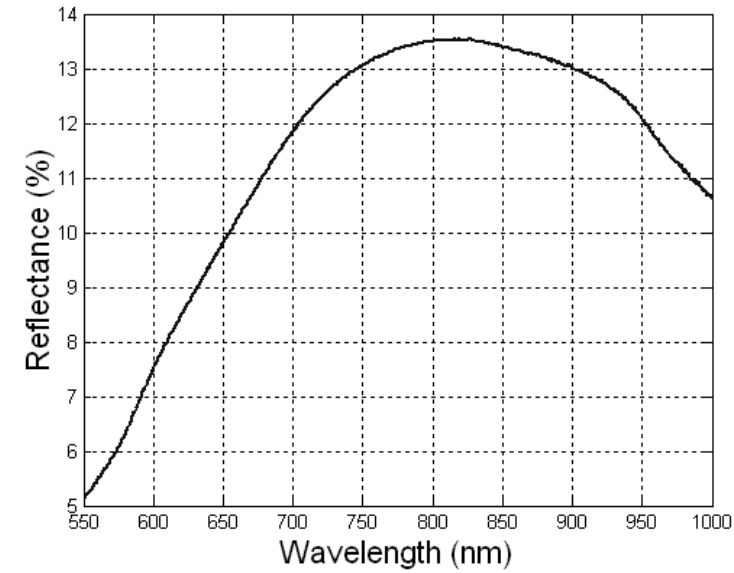

(b)

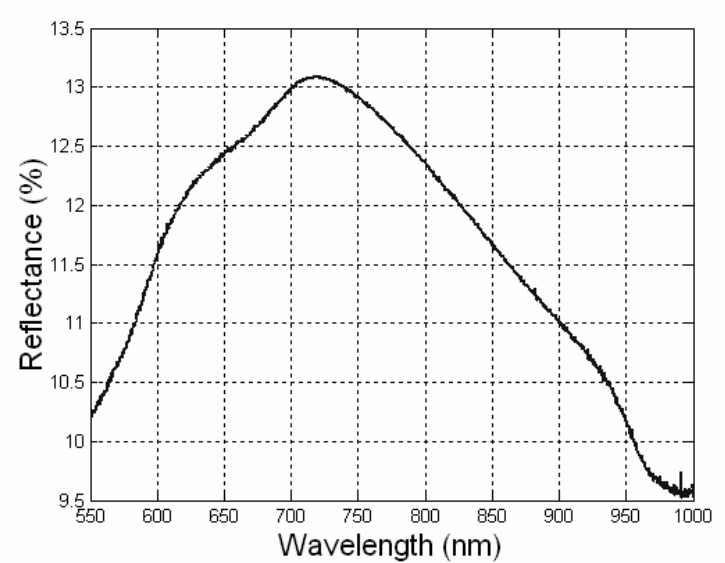

(d)

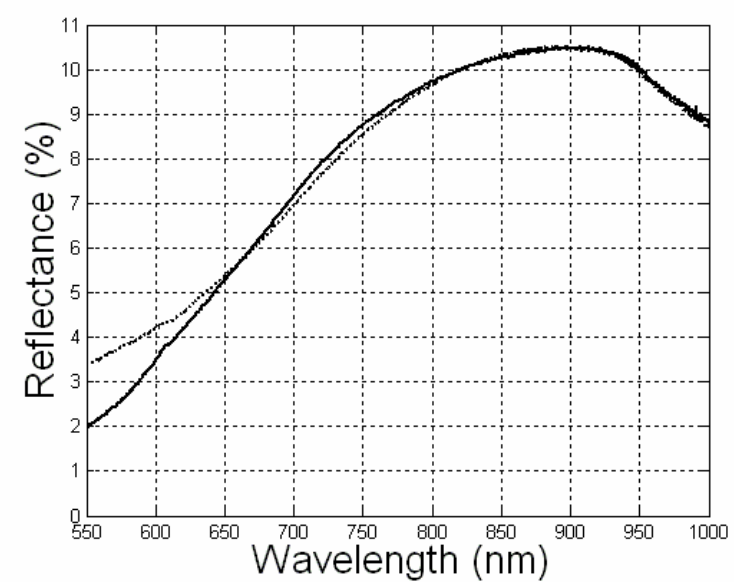

(f)

Figure 4. Reflectance spectra of (a) angioma (L5), (b) efelide (L7), (c) dermatofibroma (L8), (d) naevus nevocitic intradermal benign (L16), (e) lentigo (L15), and (f) naevus clinically atypical (-) (L14) vs. naevus nevocitic slightly atypical (----)(L9). 


\subsection{First Derivative}

To study some distinctive features and subtle differences in the slope of the spectra among lesions, the first derivatives of the lesion spectra were obtained ${ }^{16}$. In Figure 5 are shown the first derivatives averaged over an interval of 15 values centred in each wavelength, of some benign lesions compared with the first derivative of the lesions suspicious on malignancy.

As we noted previously (see 3.1.) slopes in the reflectance spectra from $550 \mathrm{~nm}$ to $800 \mathrm{~nm}$ of the non-malignant lesions are steeper than those in the suspicious of malignancy between 550-800 $\mathrm{nm}$. With the analysis of the first derivative we can see clearly these differences.

Visual assessment of the results from the Figure 5(a) shows distinctive features in the first derivative of the efelide and the naevus clinically atypical in the wavelength range between 550 and $650 \mathrm{~nm}$. The reflectance spectra of these lesions are very similar at these wavelengths. The value of the derivative is higher in the case of the efelide around $575 \mathrm{~nm}$ while the naevus clinically atypical reaches his maximum around $600 \mathrm{~nm}$, similar to the efelide, although with higher values for the atypical lesion. The pattern was similar between the two lesions in the range from $650 \mathrm{~nm}$ to $1000 \mathrm{~nm}$. In Figure 5(b) the pattern for the dermatofibroma is very different in all the wavelength range with respect to the pattern of the naevus clinically atypical. Both of them had a plateau on its curves between 625 and $700 \mathrm{~nm}$, although with different values. In the Figure 5(c) the values for the first derivative is negative beyond $720 \mathrm{~nm}$ in the case of the naevus nevocitic intradermal, while it remains positive until $875 \mathrm{~nm}$ for the clinically atypical. The curve for lentigo is also different from the atypical lesion as is shown in the Figure 5(d) in all the wavelength range.

All of the curves have similar patterns at $960 \mathrm{~nm}$, probably due to the absorption peak of the pure water and because of the high water concentration in living tissues. Despite of the similarities in that region, the first derivatives of the spectra brings, in a first approximation, clear differences and distinctive features among lesions in the spectral range from 550 to $950 \mathrm{~nm}$.

\section{DISCUSSION AND CONCLUSIONS}

Several authors ${ }^{5-10}$ have used reflectance spectroscopy for measurements of skin pigmented lesions with improved techniques. Some recent studies ${ }^{16}$ have pointed out that colour is the most important feature in distinguish melanoma from other pigmented lesions. Melanoma presents a wavelength-dependent reflectance different from other investigated lesions, in which reflectance in the infrared band appeared relevant in distinguishing between diagnostic groups. The results of our preliminary study suggest that the use of a measure system with a portable spectrometer and fiber optic probes may give valuable spectral information about different pigmented lesion. Our study look for, in the future, add some distinctive features among different skin pigmented lesions for the improvement of specificity and sensibility. We can measure over a wavelength range which has shown differences among benign and malignant lesions, and also among benign lesions. The probes used in this work cover lesions smaller than $1 \mathrm{~mm}$, so we can take spectra from different sites inside a single lesion to see subtle differences in their spectra.

It should be noted that we have just carried out a preliminary study over a small group of patients. All of them were volunteers and out of a clinical control group, so the lesions have a big variability and only two of them were lesions suspicious on malignancy. We are awaiting for the results of the histopathological analysis of this lesions, but the epiluminiscence microscopy analysis showed clearly signs of malignancy. Further processing of the collected spectral data is in course. The obtained results let to carry out in the near future a new study with a significant quantity of skin lesions. Once obtained a reflectance spectra database of both lesion and healthy skin, a statistically significant analysis of the reflectance spectra features would permit to establish algorithms and discrimination rules for a more objective differentiation of pigmented skin lesions. 


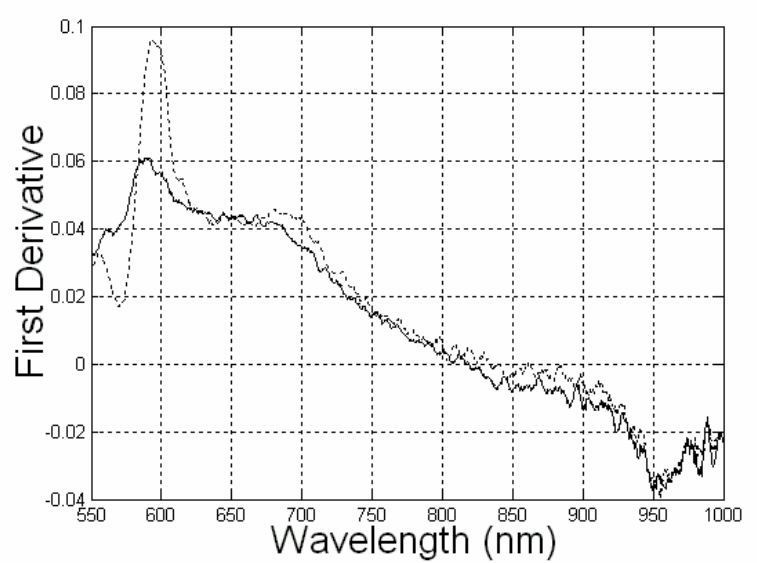

(a)

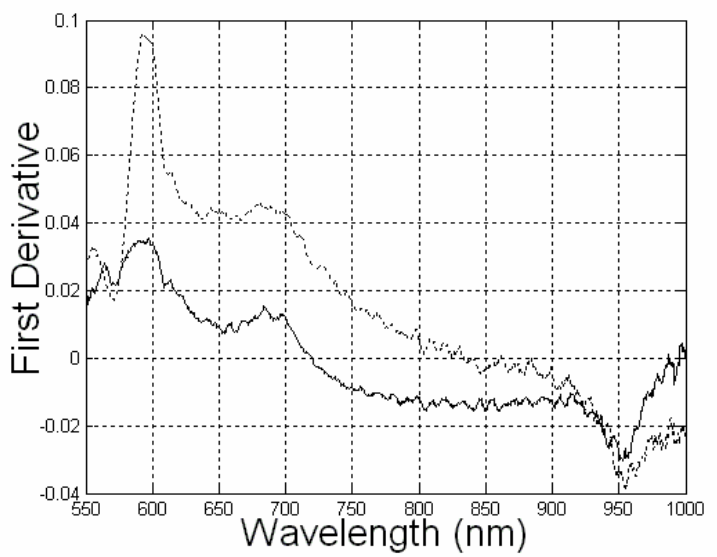

(c)

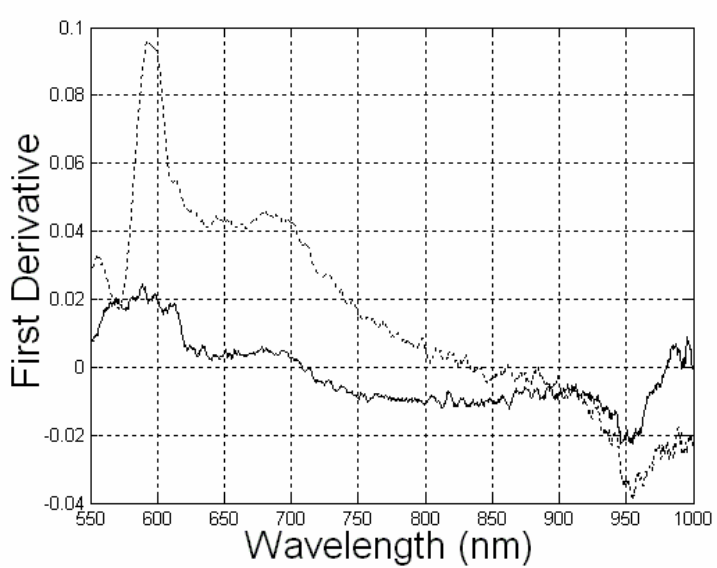

(b)

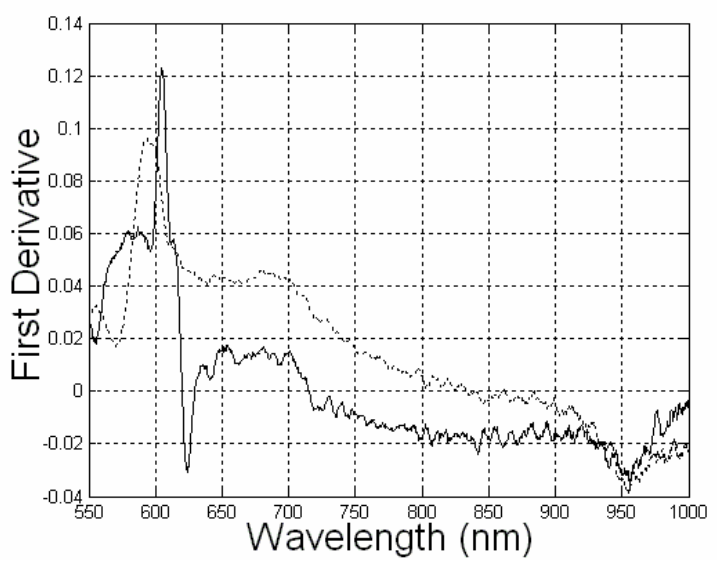

(d)

Figure 5. First derivative between 550-1000 nm obtained from (a) efelide, (b) dermatofibroma, (c) naevus nevocitic intradermal benign and (d) lentigo listed in Table 1 compared with the first derivative of the naevus clinically atypical (dashed lines).

\section{Acknowledgments}

This work has been partially supported by Spanish projects: Fondo de Investigaciones Sanitarias (FIS 01/0046-01), Universidad de Las Palmas de Gran Canaria (UNI 2002/15) and Instituto Canario de Investigación del Cáncer. MCC is a fellow from the FIS 01/0046-01 and SMLS is supported by Programa Ramón y Cajal - Spanish Ministry of Science and Technology.

\section{REFERENCES}

1. R. M. Mackie, D. Hole, J. A. A. Hunter, R. Rankin, A. Evans, K. McLaren, M. Falowfield, A. Hutcheon and A. Morris, "Cutaneous malignant melanoma in Scotland: incidence, survival and mortality, 1979-94", Br. Med. J. 315, pp. 1117-21, 1997.

2. E. Días-Rubio y J. García-Conde, Oncología clínica básica, Ediciones Arán, Madrid, 2000.

3. V. Carreño, A. Redondas y E. Cuevas, Índice UV para la población. España, Instituto Nacional de Meteorología, Madrid, 2002. 
4. Servicio Canario de Salud, Plan de salud e investigación. Análisis del estado de salud de la población y de los recursos disponibles, http://www.gobiernodecanarias.org/sanidad/scs/1/plansalud/psc02/psc02.htm.

5. R. Marchesini, M. Brambilla, C. Clemente, M. Maniezzo, A. E. Sichirollo, A. Testori, D. R. Venturoli and R. Cascinelli, "In vivo spectrophotometric evaluation of neoplastic and non- neoplastic skin pigmented lesions. I. Reflectance measurements", Photochem. Photobiol. 53, pp. 77-84, 1991.

6. R. Marchesini, N. Cascineli, M. Brambilla, C. Clemente, E. Pignoli, A. Testori, and D. R. Venturoli, "In vivo spectrophotometric evaluation of neoplastic and non- neoplastic skin pigmented lesions. II: Discriminant analysis betweeen naevus and melanoma”, Photochem. Photobiol. 55, pp. 515-522, 1992.

7. R. Marchesini, S. Tomatis, C. Bartoli, A. Bono, C. Clemente, C. Cupeta, I. Del Prato, E. Pignoli, A. E. Sichirollo and N. Cascinelli, "In vivo spectrophotometric evaluation of neoplastic and non- neoplastic skin pigmented lesions. III. CCD camera-based reflectance imaging”, Photochem. Photobiol. 62, pp. 151-154, 1995.

8. A. Bono, S. Tomatis, C. Bartoli, N. Cascinelli, C. Clemente, C. Cupeta and R. Marchesini "The invisible color of melanoma. A telespectrophotometric diagnostic approach on pigmented skin lesions" European J. Cancer 32 A, pp. 727-729, 1996.

9. S. Tomatis, C. Bartoli, A. Bono, N. Cascinelli, C. Clemente and R Marchesini, "Spectrophotometric imaging of cutaneous pigmented lesions: discriminant analysis, optical properties and histological characteristics", J. Photochem. Photobiol. B. 42, pp. 32-39, 1998.

10. A. Bono, S. Tomatis, C. Bartoli, G. Tragni, G. Radaelli, A. Maurichi. and R. Marchesini, "The ABCD system of melanoma detection. A spectrophotometric analysis of the asymmetry, border, color and dimension”, Cancer 85, pp. 7277, 1999.

11. V. P. Wallace, D. C. Crawford, P. S. Mortimer, R. J. Ott and J. C. Bamber, "Spectrophotometric assessment of pigmented skin lesions: methods and feature selection for evaluation of diagnostic performance”, Phys. Med. Biol. 45, pp. 735-751, 2000.

12. M. Moncrieff, S. Cotton, E. Claridges and P. Hall, "Spectrophotometric intracutaneous analysis: a new technique for imaging pigmented skin lesions", Br. J. Dermatology 146, pp. 448-457, 2002.

13. I. J. Bigio, T. R. Loree, J. R. Mourant, T. Shimada, K. Story-Held, R. D. Glickman, and R. L. Conn, "Optical diagnostics based on elastic scattering: recent clinical demonstrations with the Los Alamos Optical Biopsy system", Proc. SPIE 2081, pp. 174-184, 1993.

14. I. J. Bigio and J. R. Mourant, "Ultraviolet and visible spectroscopies for tissue diagnostics: fluorescence spectroscopy and elastic-scattering spectroscopy." Phys. Med. Biol. 42, pp. 803-813, 1997.

15. M. A'Amar, R. D. Ley, P. M. Ripley and I. J. Bigio, "In vivo diagnosis of chemically induced melanoma in an animal model using UV-visible and NIR elastic scattering spectroscopy: preliminary testing", Proc. SPIE 4254, pp. 144150, 2001.

16. G. Talsky, Derivative spectrophotometry. Low and higher order, VCH, New York, 1994. 